\title{
Biometría de Dientes Anteriores en Mestizos: Proporción largo/Ancho Intradental
}

\author{
Biometry of Anterior Teeth in Mestizos: Intradental Long/Wide Ratio
}

\author{
Luis Miguel Ramirez ; Luis Ernesto Ballesteros² \& Camilo José Preciado $^{3}$
}

\begin{abstract}
RAMIREZ, L. M. ; BALleSTEROS, L . E. \& PRECIADO, C. J. Biometría de dientes anteriores en mestizos: proporción largo/ ancho intradental. Int. J. Morphol., 35(3):1075-1082, 2017.

RESUMEN: Las proporciones largo/ancho intradental han sido propuestas en más de once diferentes modelos a la fecha pero no se han estudiando en población mestiza Colombiana. Se utilizaron modelos de agrupamiento jerárquico (K-Means) para entender cual explica mejor la distribución de los datos. También se analizaron co-variables de sexo, edad y atrición leve para evaluar su influencia sobre la distribución general. Fueron utilizadas fotografías estandarizadas de dientes anteriores de 274 individuos de ambos sexos con dientes completamente erupcionados y sanos. Las mediciones fueron realizadas con programas informáticos calibrados (error de 0,05 $\mathrm{mm}$ ). Se utilizó la prueba Chi Cuadrado para demostrar que las co-variables el sexo (valor de $\mathrm{p}=0,09$ ), edad (valor de $\mathrm{p}=0,54) \mathrm{y}$ atrición leve (valor de $\mathrm{p}=0,32$ ) no tuvieron impacto en la distribución de las proporciones dentales. Con respecto al análisis de conglomerados a través de las K-Means, se identificaron dos grupos diferenciados en toda la muestra: proporciones verticales (dientes mas largos) y proporciones horizontales (dientes mas anchos). Un tercer grupo solapado entre las dos tendencias lo denominamos de proporciones balanceadas. No hay un modelo de proporción intradental universal que pueda describir toda la población, pero fue posible encontrar un conjunto de modelos para los diferentes subgrupos de población. Los ideales estéticos se encuentran en abierta interpretación. Los patrones absolutos son imprácticos en biología ya que no predicen la complejidad de esta.
\end{abstract}

PALABRAS CLAVE: Proporciones intradental; Dientes anteriores; Modelos; Mestizos.

\section{INTRODUCTION}

El tamaño dental es el primer paso a considerar en el logro de una restauración balanceada que implique rehabilitar, corregir o reemplazar dientes de forma armoniosa. Las condiciones que afecten esta biometría (anodoncia, malformación, deterioro, enfermedad periodontal o la combinación de estas), siempre supone un reto para el operador en la búsqueda de su corrección y de cara a una justa proporción, basada en una identidad matemática.

La proporción morfométrica intradental (relación entre largo y ancho) de dientes anteriores es una manera de expresar la forma relativa de un diente y es un dato indispensable que facilita al periodoncista y rehabilitador oral el calculo de las dimensiones dentales (biológicas y clínicas) más apropiadas y estéticas. Procedimientos como la reubicación del margen gingival (alargamiento de corona clínica), del complejo dentogingival, busca lograr medidas balanceadas y agradables perio-dentales pero igualmente un acondicionamiento biomecánico (longitud de la pared axial), a veces necesario, en la rehabilitación oral para el logro de retención y estabilidad en el funcionamiento de estructuras protésicas fijas (Rosenberg et al., 1980; Fletcher, 2011).

En un principio, el "estándar de oro" aceptado para la relación de altura dental (desde el borde inicial hasta el borde de la encía libre), contra la anchura (limites proximales), se estimaba en un cociente del $80 \%$; sin embargo varios autores han demostrado que este cociente realmente cae en un rango, lo que supone un disenso al respecto y probablemente por las diferencias etno-geograficas de cada población estudiada. Con esto en mente, los promedios y dispersión (en paréntesis) sugeridos por cada autor son: Cooper $82 \%$ (+/- 66-96), Wolfart $75 \%$ (+/- 66-85), Parnia $83 \%(+/-72-96)$ y $82 \%(+/-71-94)$, Hasanreisoglu $81 \%$ (+/- 76-86), Olson $71 \%(+/-66-76)$, Magne $78 \%$ (Central), y $73 \%$ (Lateral y Canino), Black $76 \%$ (+/- 72-80), Chu 77-

\footnotetext{
${ }^{1}$ Profesor Titular Facultad de Odontología, Universidad de Antioquia. Medellín, Colombia.

${ }^{2}$ Profesor Titular Departamento de Ciencias Básicas, Universidad Industrial de Santander. Bucaramanga, Colombia.

${ }^{4}$ Profesor Auxiliar Facultad de Odontología, Universidad de Antioquia. Medellín, Colombia.
} 
$78 \%$ (+/- 72-81), Sterret $81 \%$, Kerr $73 \%$ y Condon et al. (2011) $88 \%$ para centrales, $77 \%$ para laterales y $80 \%$ para caninos. Stephen Chu a trasladado su proporción ideal a calibradores (Chu's Aesthetic Gauges, Hu-Friedy Inc, Chicago, IL), que buscan la planeación de procesos clínicos en los que el tamaño dental basado en la función largo/ancho del diente busca ser usada como modelo predictorio de una mayor estética y balance morfométrico dental ideal (Guillen et al., 1994; Rosenberg et al.; Sterrett et al., 1999; Magne et al., 2003; Fletcher; Chu, 2007a,b; Chu et al., 2008, 2009).

Con el propósito de buscar la distribución que mejor explica la proporción intradental en dientes anteriores de mestizos Colombianos, se analizaron sus medidas y se compararon con los medidas propuestas por otros investigadores. Un modelo de agrupamiento jerárquico en Cluster Kmeans fue desarrollado en la población estudiada para buscar subgrupos dentro de la muestra mestiza que mejor explicaran la distribución de las proporciones intradentales. Finalmente concluimos que no hay un modelo de proporción largo/ancho universal que pueda describir a toda la población, sin embargo si fue posible encontrar una tendencia a dos tipos de proporción intradental en mestizos Colombianos. Las implicaciones de sus resultados serán discutidos mas adelante.

\section{MATERIAL Y MÉTODO}

Población y medidas dentales: Se usaron fotografías estandarizadas de los dientes anteriores totalmente erupcionados y sanos. Estas fueron tomadas por un solo operador en 424 individuos de ambos sexos con un rango etáreo de 18-39 años (Promedio 23,27 +/- 4,08). El Comité de Ética de la Facultad de Odontologia de la Universidad de Antioquia aprobó la investigación. El consentimiento informado del paciente se obtuvo antes de tomar las fotografías.

Criterios de selección: Los criterios de inclusión contemplaron dientes (central, lateral y canino) con estructura incisal conservada y encías sanas con margen gingival libre por encima de la unión cemento-amelica. Los criterios de exclusión abarcaron dientes con malformaciones dentales, restauraciones en dientes anteriores, daño en la integridad del esmalte incisal (desgaste incisal, fracturas), presencia de inflamación gingival y enfermedad periodontal activa con evidente daño de la arquitectura gingival por perdida de inserción periodontal pre-existente, cirugías periodontales, presencia de hiperplasias gingivales, erupción alterada. Se descartaron 150 muestras por estar en los criterios de exclu- sión y fueron usados 274 para el estudio (44 \% mujeres y 56 $\%$ hombres). Los caninos con desgaste ligero en su prominencia incisal fueron medidos y asignados a una co-variable (atrición leve) para su análisis y correspondió al $43 \%$ de la muestra.

Estandarización fotográfica: Las fotografías fueron tomadas con retractor labial (PTJ Intl Co, Houdemont, Francia) en posición sentada y apoyo occipital manteniendo el plano de Frankfort paralelo al suelo. El operador utilizo una cámara Canon IE3 (12 megapíxeles) con macro Canon de 100 $\mathrm{mm}$ y un flash de anillo Sigma. En el plano sagital, el enfoque del lente macro se centró con dientes de línea media. Se obtuvo plano transversal paralelo a la rejilla horizontal del lente con relación al plano intercanino. El macro se utiliza en el modo manual y en proporción 1:2 que mantiene invariable la luz, registro y distancia de enfoque, lo que estandariza la comparación entre los dientes de los participantes. Para obtener la profundidad de campo en el enfoque se utilizó la mayor amplitud del diafragma.

Configuración del instrumento de medición: Las mediciones las realiza un operador con regla de Photoshop (Adobe Photoshop CS6, Adobe Systems Inc, San José, California). La regla se configura mediante una imagen de $10 \mathrm{~mm}$ de un calibrador Mitutoyo en igual proporción (2:1) a la de las fotos de los dientes. La medición digital y física fue confirmada usando algunos modelos de yeso de los participantes y como controles de las mismas medidas, hallando un error centesimal entre medidas $(0,05 \mathrm{~mm})$. Doce longitudes largo $\mathrm{y}$ ancho de cada diente superior anterior $(13,12,11,21,22$, 23) fueron registradas. Se tomaron seis horizontales (ancho) mediante el registro de la zona media incisal más ancha de cada diente (perpendicular al eje mayor) y seis verticales (largo) conforme el eje principal del diente. La razón largo/ ancho se obtiene como cociente de ambas medidas. Los datos fueron registrados en Excel (Excel, Microsoft Corp, Redmond, Washington) y se analizaron en el paquete estadístico R Project 3.2.3.

\section{RESULTADOS}

Se organizó por cuartiles la porporcion largo-ancho intradental de la muestra en la Tabla I. Se realizó análisis de la base de datos y se corroboro la correcta distribución de esta con un test de normalidad (Kolmogorov-Smirnoff). Se describieron los datos con análisis multivariante de componentes principales (ACP) y por medio de Cluster (K-means) por ser el algoritmo de clasificación que mejor explica los datos entre otros métodos también usados (Vecino mas lejano, Ward, Vecino mas cercano). Se observó una tendencia 
RAMIREZ, L. M. ; BALleSTEROS, L . E. \& PRECIADO, C. J. Biometría de dientes anteriores en mestizos: proporción largo/ancho intradental. Int. J. Morphol., 35(3):1075-1082, 2017.

Tabla I. Medidas de proporciones largo-ancho de la muestra distribuido por cuartiles para los seis dientes anteriores superiores.

\begin{tabular}{lllllll}
\hline & Min. & 1st Qu. & Median & Mean & $3^{\mathrm{d}}$ Qu. & Max. \\
\hline 23 & 0.5835 & 0.7358 & 0.7801 & 0.7893 & 0.8377 & 1.088 \\
22 & 0.5835 & 0.7358 & 0.7801 & 0.7893 & 0.8377 & 1.088 \\
21 & 0.6602 & 0.7952 & 0.8399 & 0.8405 & 0.8879 & 1.042 \\
11 & 0.6511 & 0.8073 & 0.8468 & 0.8487 & 0.8950 & 1.031 \\
12 & 0.5611 & 0.7301 & 0.7823 & 0.7903 & 0.8502 & 1.065 \\
13 & 0.5778 & 0.7362 & 0.7979 & 0.8002 & 0.8543 & 1.027 \\
\hline
\end{tabular}

Tabla II. Medidas de proporciones largo-ancho para los conglomerados 1 y 2 del Cluster K-Means.

\begin{tabular}{lllllll}
\hline Grupo & Ratio23 & Ratio22 & Ratio21 & Ratio11 & Ratio12 & Ratio13 \\
\hline 1 & 0.8402844 & 0.8402844 & 0.8896025 & 0.8990694 & 0.8625449 & 0.8628464 \\
2 & 0.7501319 & 0.7187952 & 0.8027684 & 0.8099847 & 0.7347561 & 0.75211329 \\
\hline
\end{tabular}

Tabla III. Medidas de proporciones largo-ancho para el conglomerado heterogéneo o combinado del Cluster KMeans.

\begin{tabular}{lllllll}
\hline & Ratio23 & Ratio22 & Ratio21 & Ratio11 & Ratio12 & Ratio13 \\
\hline Heterog. & 0.8185881 & 0.7572546 & 0.8464345 & 0.8579342 & 0.8129460 & 0.8190354 \\
\hline
\end{tabular}

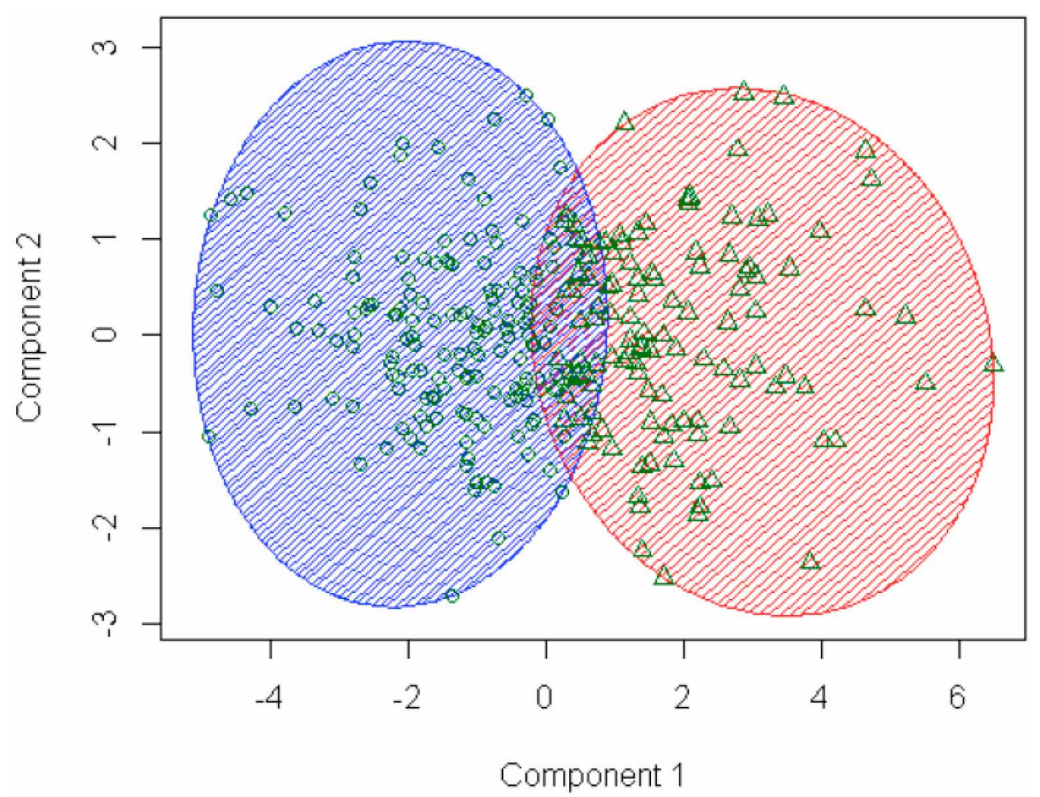

Fig 1. Análisis en Cluster con dos grupos conglomerados para la muestra estudiada. Una proporción mas ancha u horizontal explica el Cluster 1 (izquierdo) y una proporción mas alargada o vertical explica el Cluster 2 (derecho). ni explican la distribución de los conglomerados. El Test Chi-cuadrado en los clusters demuestran no ser variables dependientes (sexo $\mathrm{p}$-value $=0.09671$; atrición $\mathrm{p}$-value $=0.3201)$.

El análisis en Cluster muestra la tendencia a formar dos grupos conglomerados para la muestra estudiada (Fig. 1). Una proporción mas ancha u horizontal (mas cercana a $100 \%$ ), explica el Cluster 1 (izquierdo) y una proporción mas alargada o vertical (menor a una proporción del $100 \%$ ), explica el Cluster 2 (derecho). La Tabla II muestra la proporción para los dos grupos del K-Means. Los valores cercanos a cero representan un subgrupo combinado o de proporción balanceada ( 25 individuos, $9 \%$ de la muestra), ubicado solapadamente entre los dos conglomerados mayoritarios. La Tabla III muestra la proporción para este grupo balanceado o heterogéneo. La distribución por sexo en el Cluster 1 fue Mujeres=81, Hombres=74 y para el Cluster 2 fue Mujeres=75, Hombres=44. de los datos a formar dos grupos (máximo numero de Clusters que mejor explican los datos). Se hizo un análisis de los subgrupos formados encontrando que las co-variables edad $(\mathrm{p}$ - value $=0.5401)$, sexo y atrición no influencian
En la Figura 2 se observan diagramas de dispersión de los grupos para la muestra entera mostrando dos conglomerados definidos y pareados. Para el Cluster 1 y con un 95 $\%$ de confianza las medias pareadas fueron diferentes en los 
$0.6 \quad 0.9$
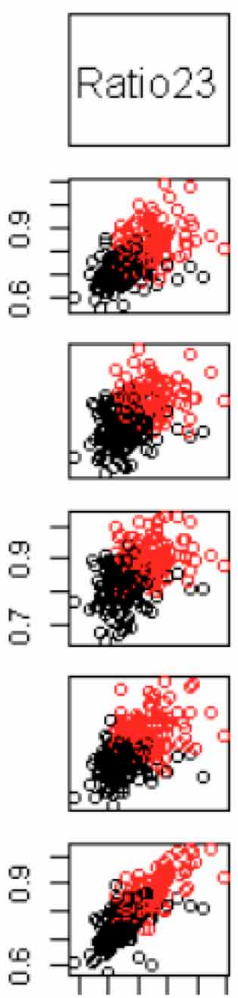

$0.6 \quad 0.9$
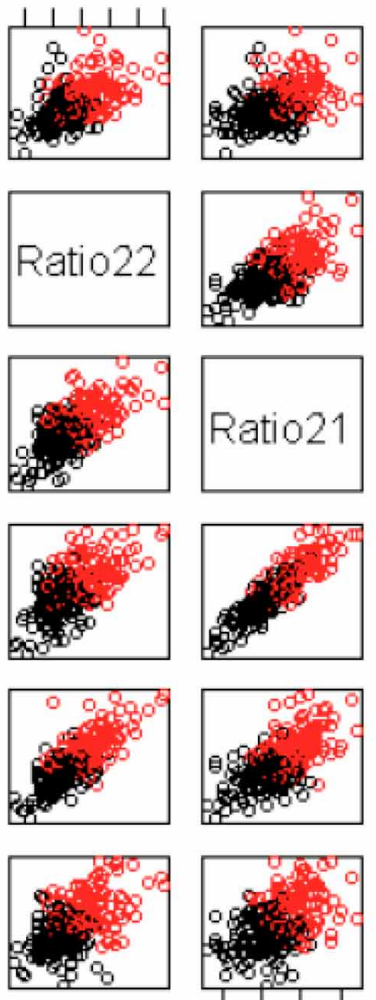

$0.7 \quad 0.9$
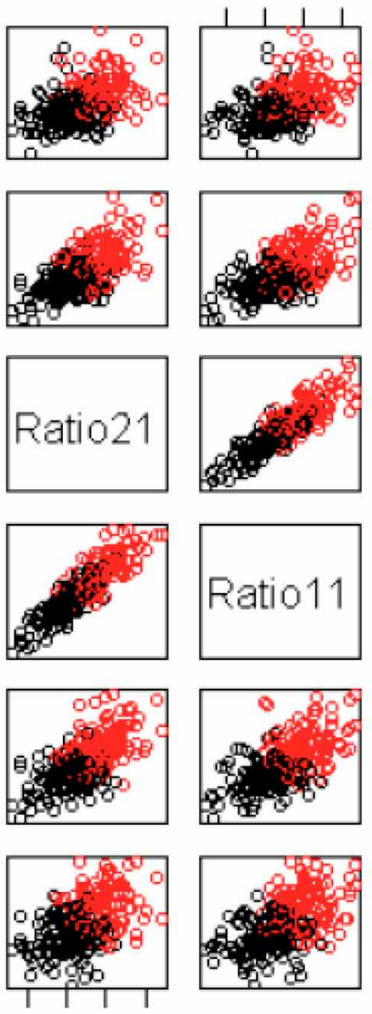

$0.7 \quad 0.9$
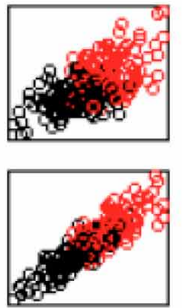

\section{Ratio11}
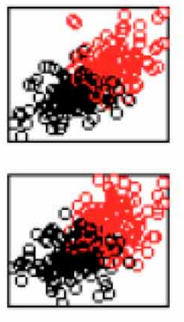

$\begin{array}{lll}0.6 & 0.8 & 1.0\end{array}$
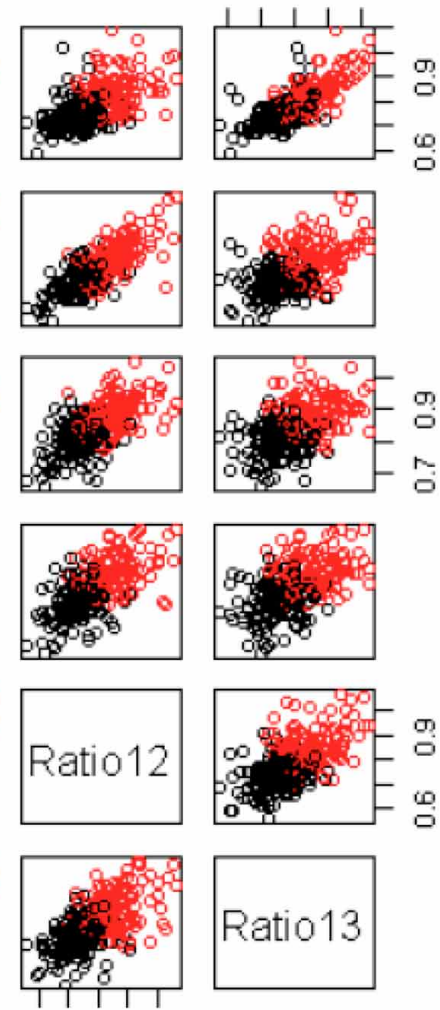

Ratio13

Fig 2. Diagrama de dispersión de los grupos para la muestra entera mostrando dos conglomerados definidos y mayormente no pareados excepto $11 / 21$.

Histograma Razon23- Cluster1

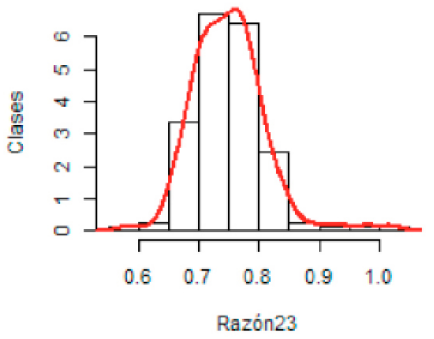

Histograma Razon11- Cluster1

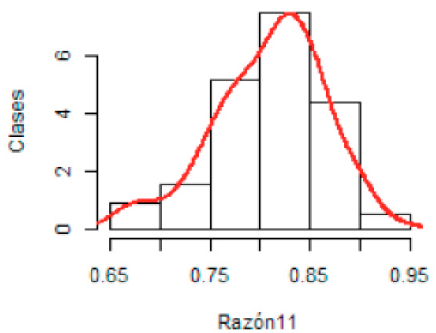

Histograma Razon22- Cluster1

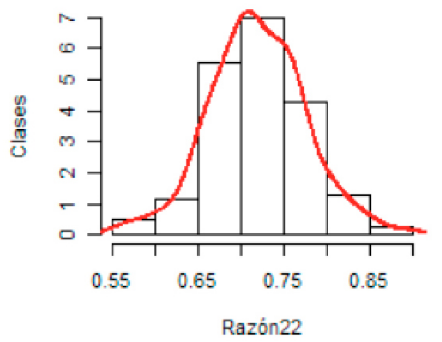

Histograma Razon12- Cluster1

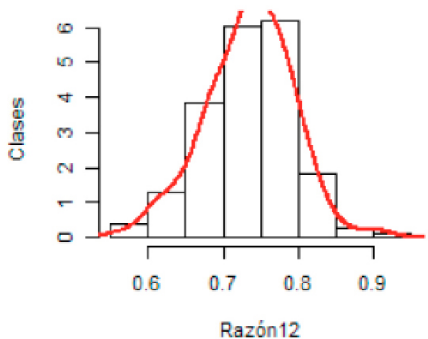

Histograma Razon21- Cluster1

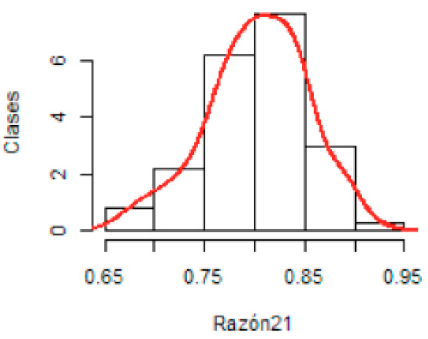

Histograma Razon13- Cluster1

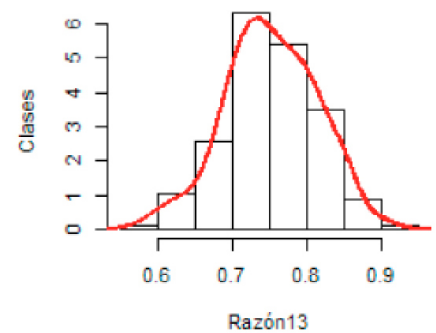

Fig 3. Histogramas de la distribución para cada diente anterior conforme el Cluster 1 encontrado en el análisis en Cluster K-Means. Eje x muestra el la proporción y eje Y la frecuencia. 
conglomerados para los dientes 23 y 13 (p-value $=0.01291)$, así como para el 22 y 12 (p-value $=0.02341)$, pero iguales para el 11 y 21 (p-value $=0.1646)$. Para el Cluster 2 las medias pareadas fueron diferentes para los dientes 22 y 12 $(\mathrm{p}$-value $=0.01517)$, pero iguales para el 11 y $21(\mathrm{p}$-value $=$ $0.2367)$, y para el 23 y 13 (p-value $=0.7725)$.
Los histogramas de la distribución para cada diente anterior $(23,22,21,11,12,13)$ conforme el Cluster 1 (Fig. 3 ) y Cluster 2 (Fig. 4) encontrados en el análisis en Cluster K-Means se muestran con un Eje x para la proporción y un eje Y para la frecuencia.
Histograma Razon23- Cluster2

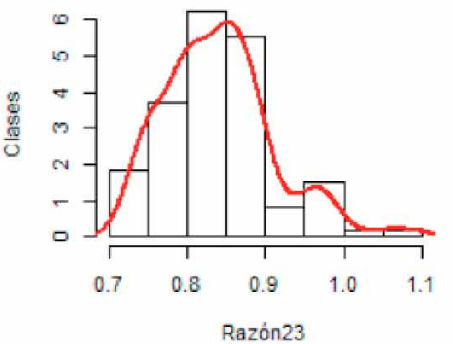

Histograma Razon11- Cluster2

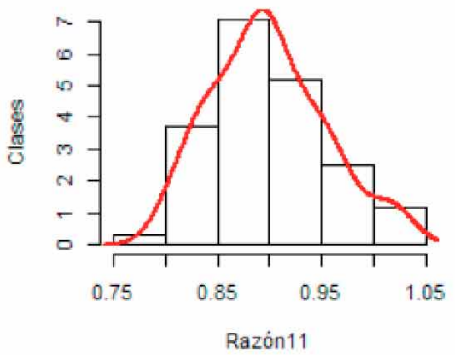

Histograma Razon22- Cluster2

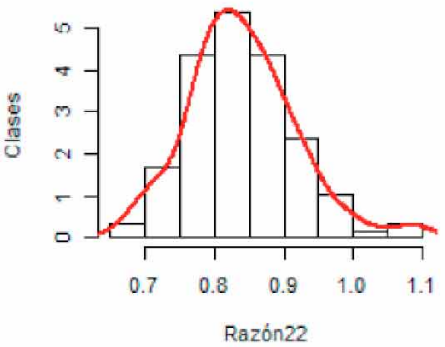

Histograma Razon12-Cluster2

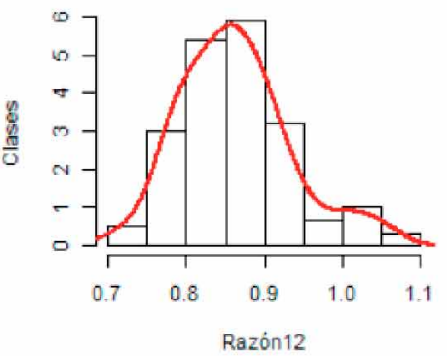

Histograma Razon21. Cluster2

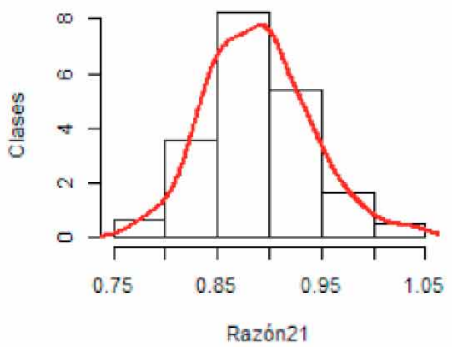

Histograma Razon13- Cluster2

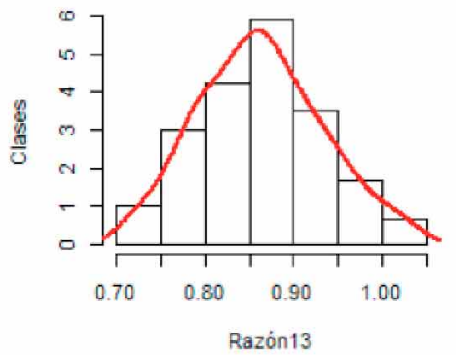

Fig 4. Histogramas de la distribución para cada diente anterior conforme el Cluster 1 encontrado en el análisis en Cluster K-Means. Eje x muestra el la proporción y eje Y la frecuencia.

Proporción Horizontal

Proporción Vertical

Proporción Balanceada

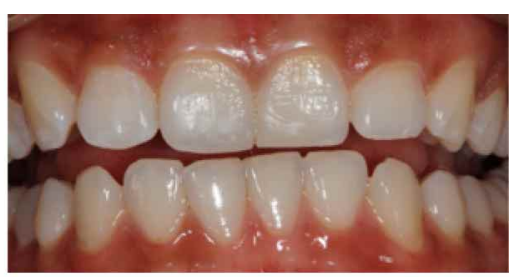

\section{DISCUSIÓN}

La oferta actual de once modelos de proporción largo/ancho intradental, supone un evidente disenso de una pretendida proporción largo/ancho universal. En nuestra muestra mestiza pudimos observar la presencia de subgrupos con una clara tendencia a una distribución de grupos correspondientes a dientes mas anchos o de proporción horizontal y a dientes mas largos o de proporción vertical (Fig. 5). Igualmente un $9 \%$ de esta población en la que las medidas estaban en una zona solapada de ambos grupos y que la denominamos como proporción balanceada. Los resultados no sorprenden debido a la mezcla étnica de poblaciones mestizas que de forma ancestral no balanceada contempla población amerindia, afro-descendiente y europea (Rosenberg et al.; Chu; Fletcher).

Fig 5. Grupos encontrados en población mestiza Colombiana: Proporción horizontal y vertical. La mezcla de ambas se considera como proporción balanceada. 
Tabla IV. Proporciones general y discriminada por diente de muestra mestiza Colombiana. Medidas para el Grupo 1, Grupo 2 y para el grupo solapado entre ambos. Se muestra el promedio de los tres.

\begin{tabular}{lcccc}
\hline Proporción/Diente & Central & Lateral & Canino & Promedio \\
\hline Grupo 1 (Horizontal) & $89 \%(+/-0,01)$ & $85 \%(+/-0,00)$ & $84 \%(+/-0,01)$ & $86 \%(+/-0,02)$ \\
Grupo 2 (Vertical) & $80 \%(+/-0,02)$ & $72 \%(+/-0,02)$ & $75 \%(+/-0,00)$ & $76 \%(+/-0,04)$ \\
Solape Grupo 1 y 2 & $86 \%(+/-0,00)$ & $78 \%(+/-0,03)$ & $81 \%(+/-0,00)$ & $82 \%(+/-0,03)$ \\
\hline
\end{tabular}

Otro factor que hace compleja la escogencia de la proporción adecuada en la población mundial es que se sugieren tres tendencias diferentes de medidas para tener en cuenta. La primera tendencia es la de autores que proponen una sola medida universal para todos los dientes como Sterret et al. (81\%) y Kerr (73\%). La segunda tendencia es la de sugerir proporciones promedio pero con un muy amplio rango o dispersión como la que ofrecen autores como Cooper $82 \%$ (+/- 66-96) con dispersión $30 \%$, Wolfart $75 \%$ (+/66-85) con dispersión $19 \%$, Hasanreisoglu $81 \%$ (+/- 7686) con dispersión $10 \%$, Olson $71 \%$ (+/- 66-76) con dispersión $10 \%$, Black $76 \%(+/-72-80)$ con dispersión $8 \%$, Chu 77-78 \% (+/- 72-81) con dispersión $9 \%$. Inclusive en esta segunda tendencia hay autores como Parnia que ofrecen dos medidas con dos rangos y dos dispersiones diferentes: $83 \%$ (+/- 72-96) con dispersión $24 \%$ y $82 \%$ (+/- 7194) con dispersión $23 \%$. Pero hay una tercera tendencia y es la de proponer el modelo conforme una proporción dental especifica como lo hacen Magne et al. y Condon et al. con proporciones individuales: Magne et al. $78 \%$ (Central), y $73 \%$ (Lateral y Canino) y Condon et al. con $88 \%$ (central), $77 \%$ (lateral) y $80 \%$ (canino).

Nuestros grupos resultantes con proporciones horizontal y vertical, categóricamente no ajustan a los modelos propuestos en ninguna de las tres tendencias (medidas absolutas, medidas con dispersión y medidas discriminadas por diente). Nuestros resultados para una medida promedio general en el Grupo 1 es de $86 \%(+/-0,02)$ y en el Grupo 2 de $76 \%(+/-0,04)$. Ahora, al discriminarlos por diente tendríamos medidas diferentes tanto en el Grupo 1 (centrales $89 \%(+/-0,01)$, laterales $85 \%(+/-0)$ y caninos $84 \%(+/-$ $0,01)$, como en el Grupo 2 (centrales $80 \%(+/-0,02)$, laterales $72 \%(+/-0,02)$ y caninos $75 \%(+/-0)$ (Tabla IV).

Pretender comparar nuestros resultados con proporciones de amplia dispersión se hace inviable por su amplio rango, lo que supone que nuestros dos grupos encontrados ajustan bien para la población mestiza Colombiana y sugieren dos grandes tendencias: proporción horizontal y proporción vertical. El criterio de complejidad K-Means genero subgrupos dentro de la muestra que explican mejor la distribución de esta, sin embargo hay una zona solapada entre los dos grupos que parece sugerir una menor incidencia de un tercer Grupo que denominamos Balanceado que podría estar tanto en el Grupo de Proporción Vertical como en el Grupo de Proporción Horizontal por su cercanía (Fig. 5).

Las proporciones intradentales largo/ancho son evidentemente un tema polémico y sin demasiado fundamento y se observan tendencias doctrinarias que confunden. Rosentiel afirma, por ejemplo, que la gente prefiere una proporción del 75-78 \% en caucásicos lo que supone dientes alargados, propios de su zona geográfica pero evidentemente no aplicable en nuestra zona geográfica. Tsukiyama et al. (2012) y Magne et al. afirman que las proporciones en centrales deben ser mayores y dominantes que en canino y este a su vez mayor a lateral lo que no pudimos ver en la totalidad de la muestra sino solo en el Grupo 2 (Rosenberg et al.).

Los métodos de análisis multivariados son más bien poco usados en morfometria. El modelo de agrupamiento jerarquico K-Means es la primera vez que se usa para este tipo de análisis, lo que podría explicar la clara tendencia en nuestro resultados pero asimismo lo abstracto de los resultados de autores anteriores con rangos muy amplios e indefinidos, ya que trataron sus datos de forma diferente.

En relación a la muestra pareada, Condon et al; afirman que no hay diferencias de lado sin embargo nosotros las encontramos en ambos grupos: caninos y laterales diferentes en Grupo 1 y lateral diferentes en Grupo 2, lo que hace mas compleja la escogencia de la proporción adecuada en la práctica. No encontramos que las co-variables edad, sexo y atrición influenciaran la distribuían de los datos. Con relación al dimorfismo sexual hay autores que si encuentran diferencias como Chu et al., Guillen et al., Condon et al., Ditch \& Rose (1972), Potter (1970) y Arya et al. (1974); sin embargo, el sexo no influyo la distribución en población mestiza Colombiana (Rosenberg et al.; Fletcher). Creemos que aunque el rango etáreo de la muestra era amplio (18-39 años $X=23,27+/-4,08)$, la tendencia de las medidas por edad era en personas jóvenes, lo que probablemente hizo que la co-variable atrición canina leve, no tuviera efectos sobre los resultados. Esto ultimo sugiere que siempre y cuando exista una leve atrición en el vértice canino, está no modifica la proporción intradental medida y escogida. 
Los resultados sugieren la no existencia de un modelo universal que pueda describir la población mundial sin discriminar esta medida por un patrón geográfico y ancestral, lo que supone que los ideales estéticos están abiertos a interpretación. En nuestra población mestiza se muestran dos grandes tendencias en el cociente entre largo y ancho, mismas que pueden ser interpretadas como proporciones opuestas que se leen como dientes largos o anchos. Consideramos que la forma de adoptar una u otra proporción en la práctica, se debe hacer sobre la medida de la proporción del central ya que en la muestra pareada, el central fue el único que coincidió con el contralateral en los dos grupos.

Consideramos que la influencia que ejerce el tamaño dental (proporcion largo/ancho) sobre los estándares estéticos y el mejor balance, sería tan abstracta como la misma belleza; sin embargo si se traslada un diente más ancho o más largo a una proporción visual ideal, evidentemente se reflejaría en una doble distribución como ya lo hemos demostrado anteriormente (Rosenberg et al.).

Reiteramos que la armonía dental no se puede percibir sin una composición completa dento-maxilo-craneo-facial en donde cada unidad hace parte de un todo. Los patrones absolutos son impracticos en biología, especialmente cuando no cuentan con su variación ni la relación multifactorial, multimodal y multicausal que rige cada una de sus partes.

\section{CONCLUSIONES}

1. No se encontró un mejor ajuste de los modelos propuestos por otros autores en población mestiza Colombiana.

2. Se observan dos tendencias de proporciones para dientes mas largos (proporción vertical) y dientes mas anchos (proporción horizontal).

3. El diente central determinaría la proporción a usar por ser el más homogéneo al estar pareado en ambos grupos.

4. Se nota una heterogeneidad en una pequeña población solapada de la muestra que representa el $9 \%$ de esta y que consideramos como una proporción balanceada.

5. Las co-variables sexo, edad y atrición no mostraron diferencias en la población general y no impactaron la distribución general de proporciones al no explicar la variabilidad de los datos ni los grupos obtenidos por los clusters.

6. Se encontraron diferencias de lado para laterales y cani- nos pero no dimorfismo sexual.

7. Los ideales estéticos se encuentran en abierta interpretación.

\section{AGRADECIMIENTOS}

Al Dr. Juan David Ospina y su semillero del programa de Estadística de la Universidad de Antioquia, Cristian Camilo Hidalgo García, por el tratamiento de los datos. No hay conflicto de intereses declarado.

RAMIREZ, L. M. ; BALLESTEROS, L . E. \& PRECIADO, C. J. Biometry of anterior teeth in mestizos: intradental long / wide ratio. Int. J. Morphol., 35(3):1075-1082, 2017.

SUMMARY: The intradental length / width ratios have been proposed in more than eleven different models to date. However they have not been studied in the Colombian Mestizo population. Hierarchical clustering models (K-Means) were used to understand which best explains the distribution of the data. Covariables of sex, age, and mild attrition were also analyzed to assess their influence on overall distribution. Standardized photographs of anterior teeth of 274 individuals of both sexes with fully erupted and healthy teeth were used. Measurements were taken with calibrated software (error of $0.05 \mathrm{~mm}$ ). Chi square test was used to show that the co-variables sex $(\mathrm{p}$ value $=0.09)$, age $(\mathrm{p}$ value $=$ 0.54 ) and mild attrition ( $p$ value $=0.32$ ) had no impact In the distribution of dental proportions. With respect to the analysis of conglomerates through the K-Means, two distinct groups were identified throughout the sample: Vertical proportions (longer teeth) and horizontal proportions (wider teeth). A third group overlapping the two trends we called balanced proportions. There is no universal intraday proportion model that can describe the entire population, but it was possible to find a set of models for different population subgroups. Aesthetic ideals are in open interpretation. Absolute patterns are impractical in biology because they do not predict the complexity of biology.

KEY WORDS: Intradental proportions; Anterior teeth; Models; Mestizos.

\section{REFERENCIAS BLIOGRÁFICAS}

Arya, B. S.; Savara, B. S.; Thomas, D. \& Clarkson, Q. Relation of sex and occlusion to mesiodistal tooth size. Am. J. Orthod., 66(5):479-86, 1974.

Chu, S. J. \& Hochman, M. N. A biometric approach to aesthetic crown lengthening: part I--midfacial considerations. Pract. Proced. Aesthet. Dent., 20(1):17-24, 2008.

$\mathrm{Chu}, \mathrm{S}$. J. A biometric approach to predictable treatment of clinical crown discrepancies. Pract. Proced. Aesthet. Dent., 19(7):401-9, 2007 a. 
Chu, S. J. Range and mean distribution frequency of individual tooth width of the maxillary anterior dentition. Pract. Proced. Aesthet. Dent., 19(4):209-15, 2007b

Chu, S. J.; Fletcher, P. D. \& Mieleszco, A. J. Clinical application of innovative measurement gauges for predictable correction of tooth size/ proportion and gingival architecture discrepancies. Quintessence Dent. Technol., 32:63-76, 2009

Condon, M.; Bready, M.; Quinn, F.; O'Connell, B. C.; Houston, F. J. \& O'Sullivan, M. Maxillary anterior tooth dimensions and proportions in an Irish young adult population. J. Oral Rehabil., 38(7):501-8, 2011.

de Castro, M. V.; Santos, N. C. \& Ricardo, L. H. Assessment of the "golden proportion" in agreeable smiles. Quintessence Int., 37(8):597-604, 2006.

Delgado-Burbano, M. E. Population affinities of African Colombians to Sub-Saharan Africans based on dental morphology. Homo, 58(4):32956, 2007.

Ditch, L. E. \& Rose, J. C. A multivariate dental sexing technique. Am. J. Phys. Anthropol., 37(1):61-4, 1972.

Fletcher, P. Biologic rationale of esthetic crown lengthening using innovative proportion gauges. Int. J. Periodontics Restorative Dent., 31(5):52332, 2011.

Guillen, R. J.; Schwartz, R. S.; Hilton, T. J. \& Evans, D. B. An analysis of selected normative tooth proportions. Int. J. Prosthodont., 7(5):410-7, 1994.

Hasanreisoglu, U.; Berksun, S.; Aras, K. \& Arslan, I. An analysis of maxillary anterior teeth: facial and dental proportions. J. Prosthet. Dent., 94(6):530-8, 2005.

Hasanreisoglu, U.; Berksun, S.; Aras, K. \& Arslan, I. An analysis of maxillary anterior teeth: facial and dental proportions. J. Prosthet. Dent., 94(6):530-8, 2005.

Huang, S. Y.; Kang, T.; Liu, D. Y.; Duan, Y. Z. \& Shao, J. L. Variability in permanent tooth size of three ancient populations in Xi'an, northern China. Arch. Oral Biol., 57(11):1467-73, 2012.

Magne, P.; Gallucci, G. O. \& Belser, U. C. Anatomic crown width/length ratios of unworn and worn maxillary teeth in white subjects. J. Prosthet. Dent., 89(5):453-61, 2003.

Potter, R. B. Dentoesthetics thru visual aids. J. Tenn. State Dent. Assoc., 50:19-22, 1970 .

Ramirez, L. M.; Ospina, J. D. \& Ballesteros, L. E. Mestizo anterior teeth's proportions. Int. J. Morphol., 34(1):223-31, 2016.

Rosenberg, E. S.; Garber, D. A. \& Evian, C. I. Tooth lengthening procedures. Compend. Contin. Educ. Gen. Dent., 1(3):161-72, 1980.

Scott, G. R. \& Turner, C. G. The Anthropology of Modern Human Teeth: Dental Morphology and its Variation in Recent Human Populations (Cambridge Studies in Biological and Evolutionary Anthropology). Cambridge, Cambridge University Press, 1997.

Sterrett, J. D.; Oliver, T.; Robinson, F.; Fortson, W.; Knaak, B. \& Russell, C. M. Width/length ratios of normal clinical crowns of the maxillary anterior dentition in man. J. Clin. Periodontol., 26(3):153-7, 1999.

Tsukiyama, T.; Marcushamer, E.; Griffin, T. J.; Arguello, E.; Magne, P. \& Gallucci, G. O. Comparison of the anatomic crown width/length ratios of unworn and worn maxillary teeth in Asian and white subjects. $J$. Prosthet. Dent., 107(1):11-6, 2012.

\author{
Dirección para correspondencia: \\ Prof. Luis Miguel Ramirez. \\ Laboratorio QST (225) \\ Facultad de Odontología \\ Calle 64 \# 52-59 \\ Universidad de Antioquia. \\ Medellín \\ COLOMBIA
}

E-mail: miguelramo.ramirez@udea.edu.co

Recibido : 14-02-2017

Aceptado: 02-05-2017 